\title{
A Design of Functional Layer with Robust Constitutive Parameters for Multilayer Metamaterials
}

\author{
Zhijie Gong, ${ }^{1,2}$ Chao Wu, ${ }^{1,2,3}$ Quan Li, ${ }^{1,2}$ Zeyong Wei, ${ }^{1,2,3}$ and Hongqiang Li $^{1,2,3}$ \\ ${ }^{1}$ School of Physics Science and Engineering, Tongji University, Shanghai 200092, China \\ ${ }^{2}$ Shanghai Key Laboratory of Special Artificial Microstructure Materials and Technology, Tongji University, Shanghai 200092, China \\ ${ }^{3}$ The Institute of Dongguan-Tongji University, Dongguan, Guangdong 523808, China
}

Correspondence should be addressed to Chao Wu; chaowu@tongji.edu.cn

Received 28 April 2017; Revised 13 August 2017; Accepted 20 August 2017; Published 25 September 2017

Academic Editor: Pavel Lejcek

Copyright (C) 2017 Zhijie Gong et al. This is an open access article distributed under the Creative Commons Attribution License, which permits unrestricted use, distribution, and reproduction in any medium, provided the original work is properly cited.

\begin{abstract}
We propose a functional layer design with robust effective parameters for multilayer metamaterial. The functional layer is consisting of two identical dielectric material layers and one layer of metallic structures sandwiched in between. The symmetric design ensures that, following standard retrieval technique, effective parameters retrieved for a single functional layer in vacuum can be used to characterize its electromagnetic contribution when stacked in a multilayer system. When applied to the fishnet structures, effective parameters of the symmetric functional layer system show great robustness against the varying of the number of layers. The symmetric functional layer design is also investigated on multilayer metamaterials consisting of several layers of different kinds of metallic structures. Transmission and reflection spectra are obtained for real structures and their effective models by finitedifferential-time-domain simulation and transfer matrix method calculation, respectively. It turns out that the effective model shows great equivalency to the real structures, and the effective parameters of symmetric functional layer design are robust at both normal and oblique incident cases. Our work provides a practical approach to design and characterize multilayer metamaterials with the well-known effective parameters retrieval technique.
\end{abstract}

\section{Introduction}

Metamaterial (MM) refers to a kind of artificial materials with subwavelength-sized embedded metal resonators which gives nearly any value of constitutive parameters $(\varepsilon, \mu)$. Such engineered media can offer novel electromagnetic properties beyond natural materials, such as negative refraction [1-3], perfect lens [4], invisible cloaking [5], and perfect absorption [6]. To realize these exotic properties in practical applications, design and fabrication of MMs are marching towards being three-dimensional [7]. It is an appropriate and simple way to construct 3D MM structures by stacking single layer structures [8-15], as planar metal structure could be easily fabricated by mature technologies such as electron beam lithography $[9,13,14,16-20]$, membrane projection lithography [21], direct laser writing [22-24], and electroplating [25]. For a MM slab with finite thickness, effective permittivity and permeability can be retrieved from reflection and transmission coefficients, known as effective parameter retrieval technique $[26,27]$. Later improvements generalized this technique to oblique incidence [28] and chirality [11, 2935] case, allowing a complete characterization of MMs.

Since single layer structures can be described with their effective constitutive parameters, the design and characterization of multilayer MMs should be easily accomplished based on the effective parameter retrieval technique. However, the retrieved parameters are directly related to a particular field, and the interaction between building blocks from different layers is inevitable in a multilayer MM. Thus, effective parameters obtained for single layer structure in vacuum cannot characterize its contribution when this layer is stacked in multilayer system. In other words, the effective parameters are not robust. For example, previous research on multilayer fishnet structure [12, 36-38] point out that, due to the coupling between different layers, effective parameters change as the number of layers increased. Though 
the effective parameters could eventually converge when sufficient number of layers is acquired, the convergence results are usually quite different to those of monolayer fishnet. Consequently, it is impractical to estimate properties of multilayer MMs with the effective parameters of single layer structure, even if all of the stacking layers are the same. Moreover, for multilayer MMs consisting of several layers of different metal structures, the coupling between different layers would be much more complex, making the effective parameters of single layer almost useless in multilayer MMs design.

In this paper, we propose a symmetric functional layer design for multilayer MMs. The functional layer is comprised of two identical dielectric layers and one layer of metallic structure sandwiched between them. We find that effective parameters of these symmetric functional layers are robust against the change of surrounding. Effective parameters of multilayer fishnet structure apply this symmetric functional layer design almost immune from the number of layers varying. Furthermore, the robustness of effective parameters is also intact in multilayer MMs consisting of several symmetric functional layers of different metallic structures at both normal and oblique incidence cases. Therefore, this symmetric functional layer design offers a practical approach to construct multilayer MMs with the well-known effective parameters retrieval technique $[26,28,39]$.

\section{Symmetric Functional Layer}

In Figure 1(a), we present schematic graph of a multilayer fishnet structure. The periods along the lateral plane are set to be $p x=p y=4 \mathrm{~mm}$, and the square holes of fishnet have widths of $b=3.6 \mathrm{~mm}$. The thickness of the metallic fishnet structures is $18 \mu \mathrm{m}$. The metal we used for fishnet structure and all other metallic structures in this paper is silver with the electric conductivity of $6.3 \times 10^{7} \mathrm{~S} / \mathrm{m}$. The dielectric spacers are made of FR 4 (orange, $\varepsilon_{\text {real }}=4.3$, loss tangent of 0.025 ) with the thickness $\mathrm{hf}=1.6 \mathrm{~mm}$. The propagation direction is along the $z$-axis and perpendicular to the surface of the fishnet. In the following discussion, the number of metal structure layers $N$ is used to specify the multilayer fishnet structures with different actual layers. As an example, the two-layer slab contains 2 metal fishnet layers.

For a homogeneous material slab, the impedance and refractive index (or the permittivity and permeability) does not depend on its thickness. In contrast, MMs must acquire a number of layers to achieve convergence of electromagnetic properties and qualify as bulk material $[7,12]$. Due to the interaction between building blocks from different layers, effective parameters vary as the number of layers increases. Generally speaking, effective parameters of monolayer structures are not as robust. In Figure 1(c), we present the retrieved results for the real part of effective refractive index $n$ for fishnet structures with different number of layers $(N=2$, $3,4,5)$. Notice that effective parameters gradually converge as the number of layers increases. However, the convergence results are completely different from results given by the 2layer structure. These features were reported in many prior studies $[7,12,28]$.
The interactions of metallic structures and EM waves are the essentials of the exotic electromagnetic properties of MMs. Though the fishnet structures from each layer are exactly the same in geometric parameters, the surrounding environments are not always the same. As shown in Figure 1(b), while the inner metallic fishnet layers are located symmetrically between two dielectric spacers, the two outmost layers of metallic fishnet are sandwiched between air and dielectric spacer, which is clearly asymmetric. Intuitively, when interacting with electromagnetic waves, the induced electromagnetic fields for inner and outmost metallic fishnet structures are expected to be symmetric and asymmetric, respectively. Thus, the resonant features are different for two kinds of fishnet structures. For $N=2$ fishnet system, both of the two fishnet layers are asymmetric; the electromagnetic behavior should be purely dominate by asymmetric layers. While the number of layers increases, the symmetric layers gradually became the majority. Correspondingly, the electromagnetic behavior gradually changed to be dominated by symmetric layers, and in other term, the retrieval parameters converge. Furthermore, the interaction between them could also induce the hybridization of resonance modes, which is shown as the ripples in effective parameter diagrams.

These properties are examined in a simple multilayer system composed of two kinds of homogeneous medium slabs. The thickness of each layer is $1.6 \mathrm{~mm}$, while the constituted parameters of two media are set to be $\varepsilon_{\mathrm{A}}=2, \mu_{\mathrm{A}}=1$ (orange) and $\varepsilon_{\mathrm{B}}=16+i, \mu_{\mathrm{B}}=1.5+0.1 i$ (blue), respectively. As shown in Figure $1(\mathrm{~d})$, the multilayer system is arranged in a BA$(\mathrm{ABA})^{m}-\mathrm{AB}$ pattern, which has inside symmetric ABA blocks and outside asymmetric $\mathrm{AB}$ and $\mathrm{BA}$ blocks. Comparing with multilayer fishnet structure, the B layers are corresponding to the metallic fishnet structure layers, and building blocks with two A layers are corresponding to the dielectric spacers. Despite the fact that no resonant structure is included, this system holds the geometric essence of the fishnet structure shown in Figure 1(a). The calculated effective refractive index for multilayer system with different number of B layers is shown in Figure 1(e). It is clear that this simplified system shows similar convergence and hybridization features as the fishnet system.

At this state, we can concluded that the conventional assembly manner of multilayer MMs causes the resonance features of the same structures to differ depending on their location and introduces interactions between different layers which result in the change of electromagnetic behavior as the number of layers increases and poor robustness of the effective parameters of monolayer structures. To overcome this problem, we proposed a symmetric functional layer design, which consists of two identical dielectric media layers and one layer of metallic structure sandwiched in between. Figure 2(a) shows sketch of a unit cell of the symmetric fishnet functional layer. The geometric parameters of metallic fishnet structure are the same as those in Figure 1(a). Two dielectric layers are made of FR4 also, and each has the thickness of $\mathrm{df}=$ $1 / 2 \mathrm{hf}=0.8 \mathrm{~mm}$, which ensures that the thickness of dielectric spacer between two adjacent metallic structures are the same as in Figure 1(a). Schematic of a 5-layer structure applying this design is shown in Figure 2(b). Obviously, all the metallic 


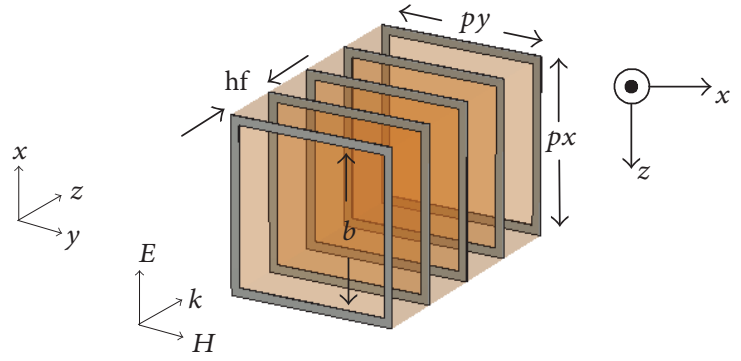

(a)

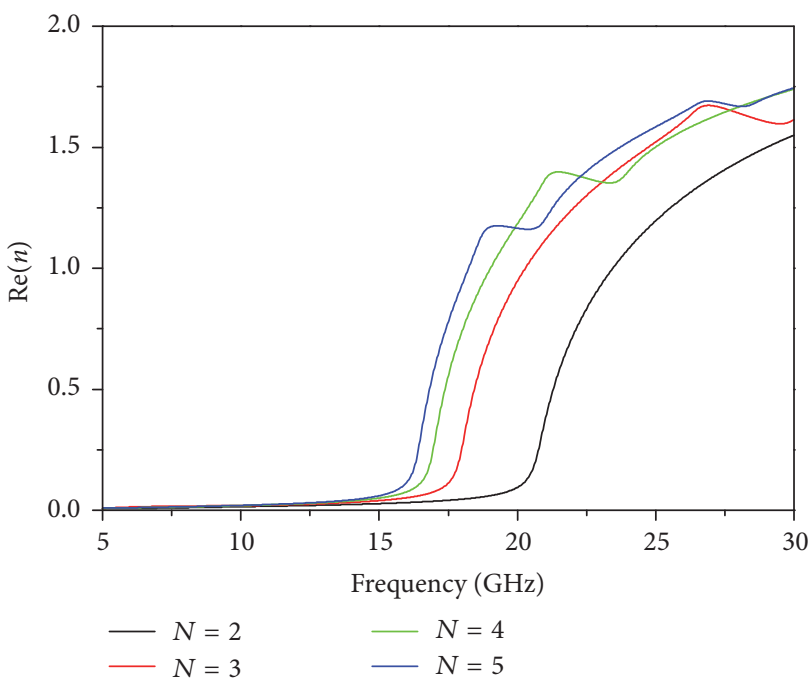

(c)

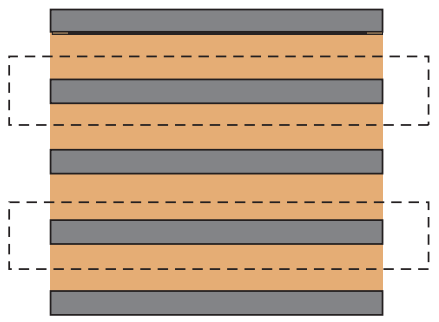

(b)
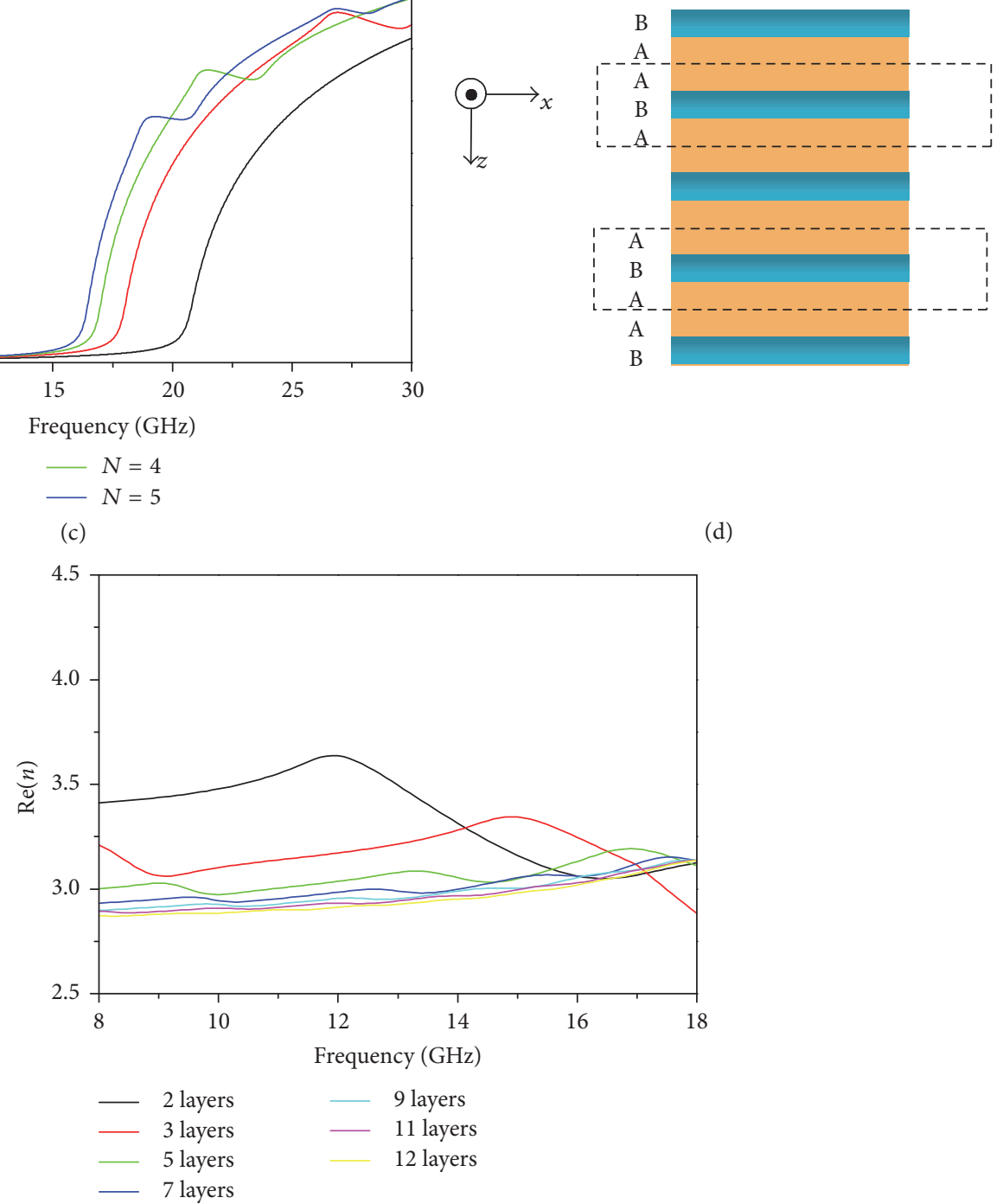

(d)

(e)

Figure 1: (a) and (b) Schematic of a conventional fishnet structure with 5 metallic layers. (c) Real part of the effective index for 2-layer, 3-layer, 4-layer, and 5-layer fishnet structures retrieved from the simulated scattering parameters. (d) Sketch of corresponding simplified multilayer system. (e) Real part of the retrieved effective index for simplified multilayer systems contains 2 layers, 3 layers, 4 layers, 7 layers, 9 layers, 11 layers, and 14 layers B medium. 


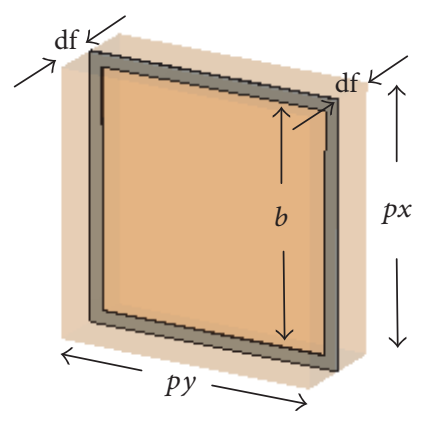

(a)

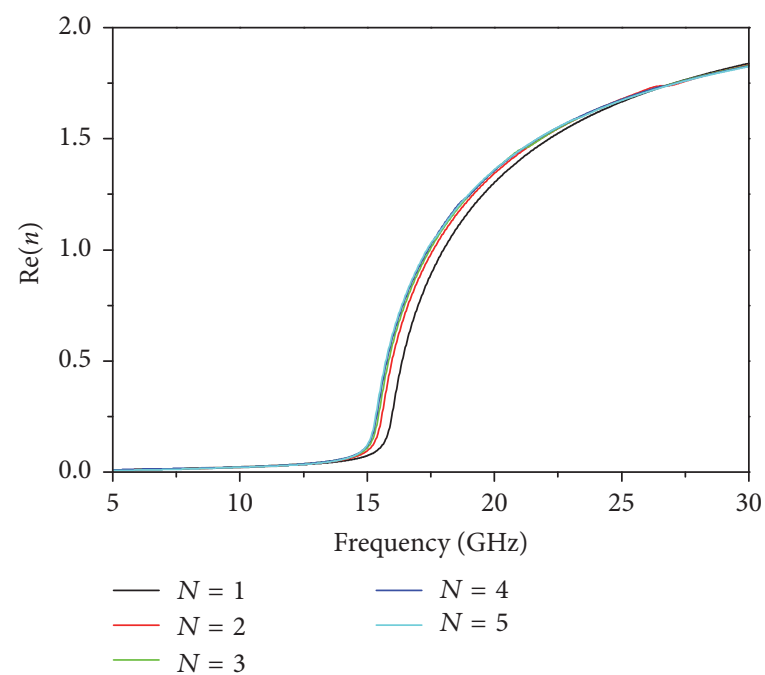

(c)

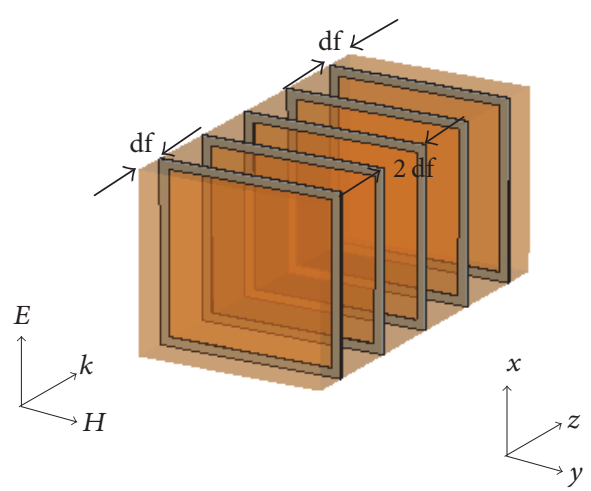

(b)

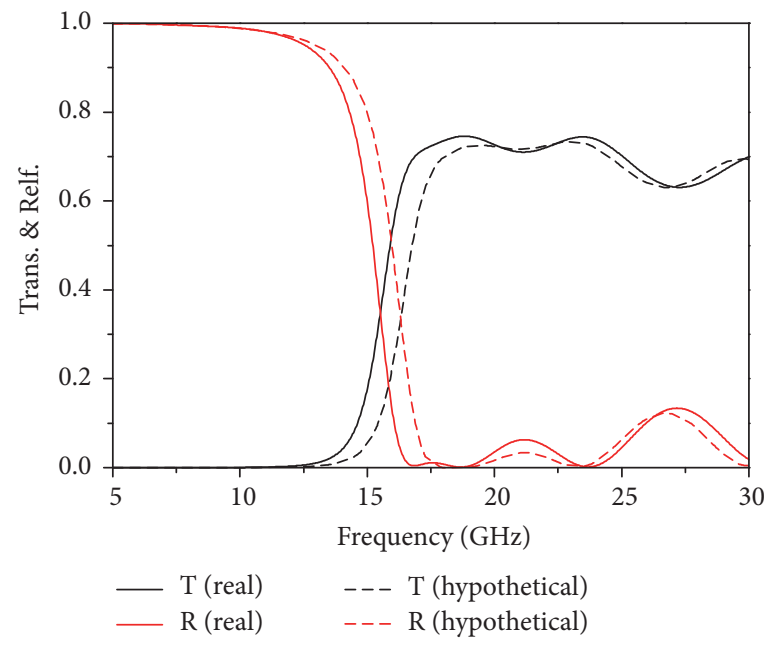

(d)

FIGURE 2: (a) The schematic picture of a unit cell of symmetric fishnet functional layer and (b) sketch of a unit cell of 5-functional-layer fishnet structures. (c) The real part of effective index for 2-functional-layer, 3-functional-layer, 4-functional-layer, and 5-functional-layer fishnet structures retrieved from the simulated scattering parameters. (d) Transmission and reflection spectra of real 5-functional-layer fishnet slab and the hypothetical homogenous slab with the thickness of 5-layer fishnet structure and the effective parameters of one-layer structure. The results are calculated for normal incidence of a plane wave, and the spectra are normalized to the intensity of the incident wave.

structure layers are equally located in the same symmetric environment. Compared to the conventional design, the modification is relatively minor that only two dielectric layers are added to the top and bottom. Consequently, the effect on the electromagnetic behavior is also little. The retrieval indexes of such multilayer system with different number of functional layers are shown in Figure 2(c). Clearly, the results are almost unchanged, and hybridization of resonant modes is also eliminated since resonant features of each functional layer are the same. This indicates that effective parameters of the symmetric fishnet functional layer are robust. To further estimate robustness of effective parameters, we introduce a hypothetical homogenous medium slab with the thickness of 5-functional-layer fishnet structure and the effective parameters of one-functional-layer structure. The transmission and reflection spectra of 5-functional-layer fishnet structure and the hypothetical slab are shown in Figure 2(d). The results agree with each other very well in a relatively wide frequency range.
In our opinion, the underlying mechanism for the robustness of our symmetric functional layer design is twofold. First, symmetric design ensures resonant features unaffected when assembled in multilayer systems. Second, different functional layers are essentially interacting through the evanescent scattering field component around metal structures. Two identical dielectric layers provide the space for them to damp down, further reducing the coupling between metallic structures, which results in retrieval parameters robust being against the varying of the number of functional layers. Furthermore, we investigated the dependence of the robustness of effective parameters on the dielectric spacer in fishnet structures. Compared to the structures in Figure 2(a), we varied dielectric layer thickness of the symmetric fishnet functional layer to thicker ( $\mathrm{df}=1.0 \mathrm{~mm}$, orange) and thinner ( $\mathrm{df}=0.5 \mathrm{~mm}$, orange) cases, and all other parameters are fixed to their respective values in Figure 2(a). Retrieval indexes of both multilayer systems with different number of functional layers are shown in Figures 3(a) and 3(c). It is clear that 


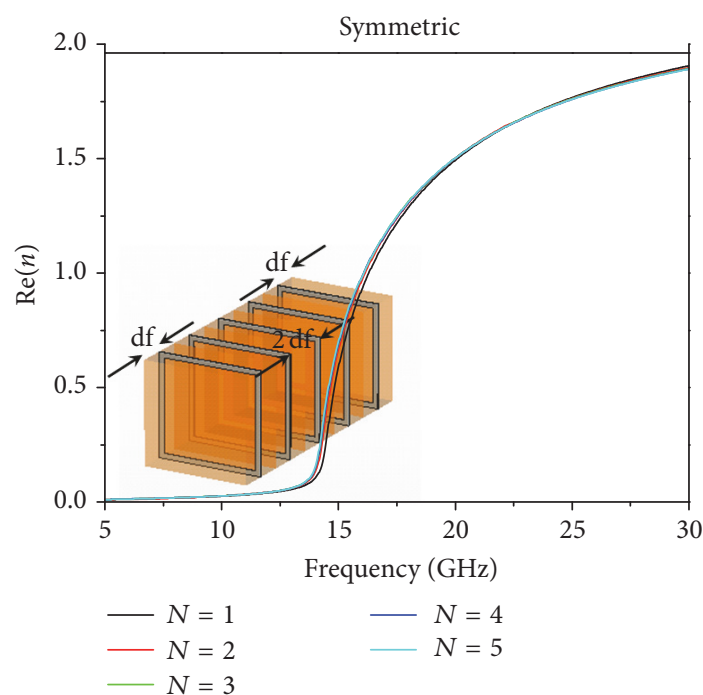

(a)
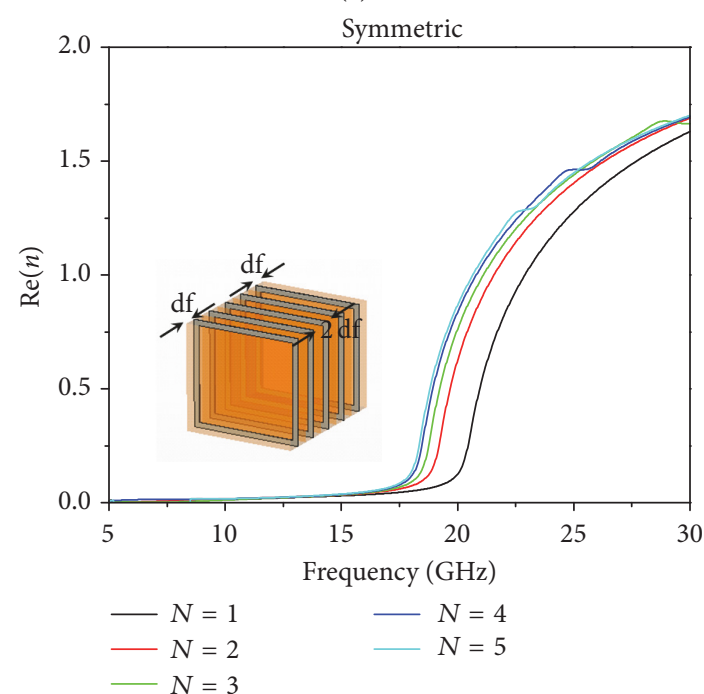

(c)

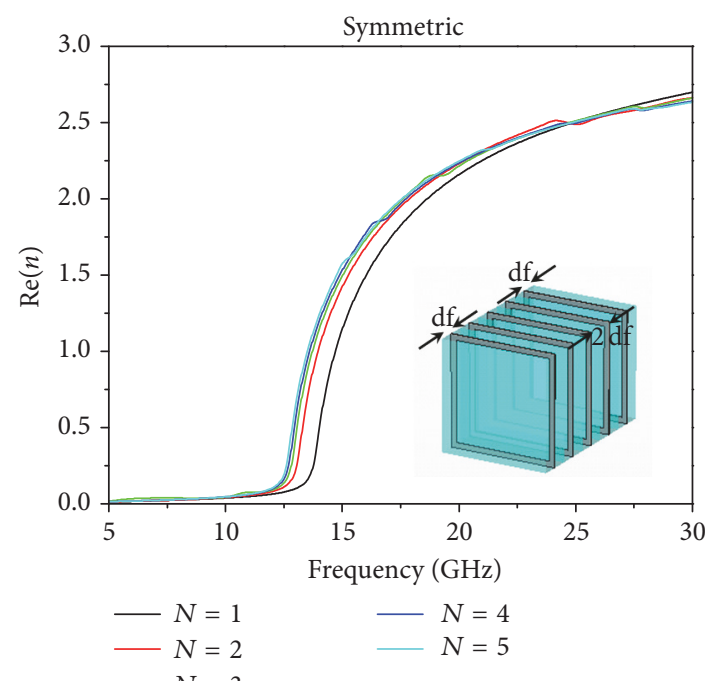

(e)

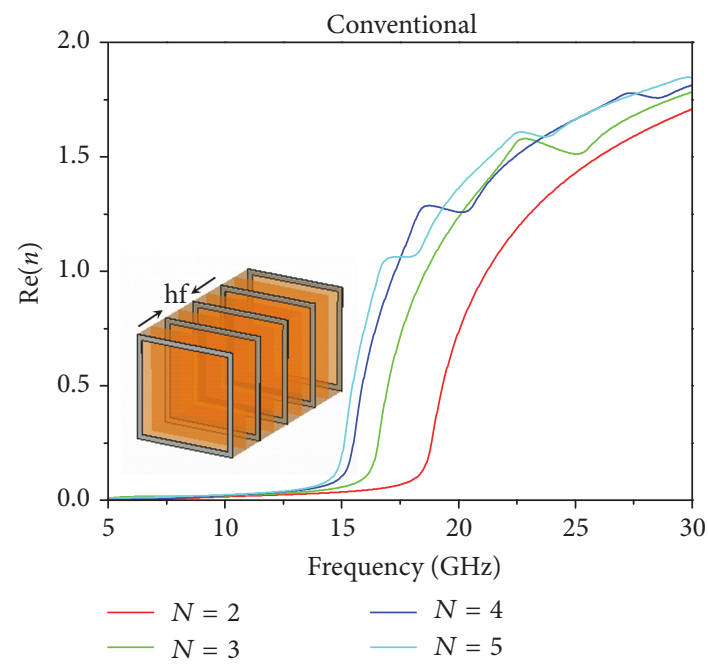

(b)

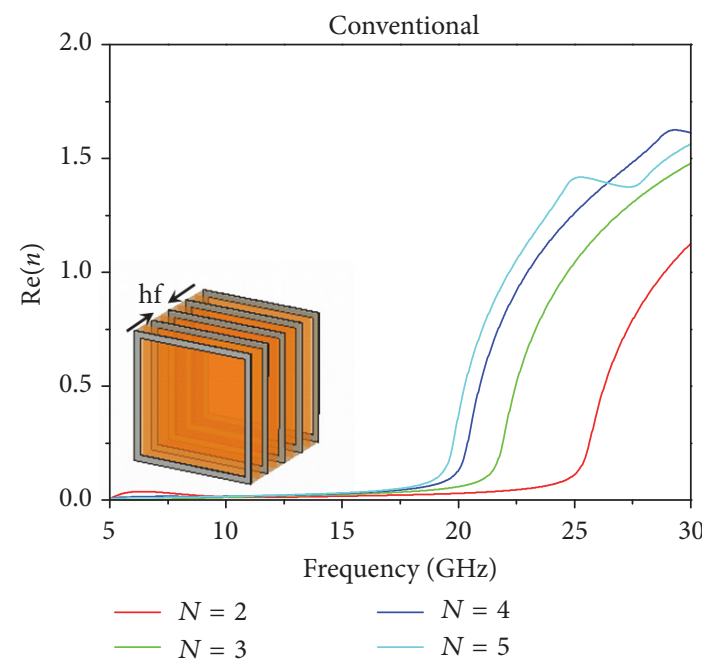

(d)

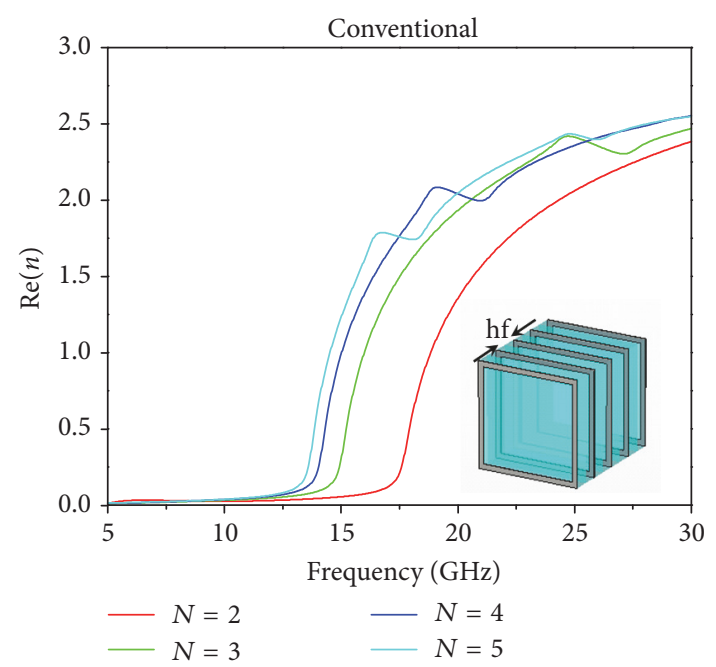

(f)

Figure 3: (a), (c), and (e) The real part of effective index for 1-, 2-, 3-, 4-, and 5-symmetric-functional-layer fishnet structures retrieved from the simulated scattering parameters with the different parameter: (a) $\mathrm{df}=1.0 \mathrm{~mm}, \varepsilon=4.3$; (c) $\mathrm{df}=0.5 \mathrm{~mm}, \varepsilon=4.3$; (e) $\mathrm{df}=0.6 \mathrm{~mm}, \varepsilon=8$. (b) (d) and (f) real part of the effective index for 2-, 3-, 4-, and 5-layer conventional fishnet structures retrieved from the simulated scattering parameters with the different parameter: (b) $h f=2.0 \mathrm{~mm}, \varepsilon=4.3$; (d) $\mathrm{hf}=1.0 \mathrm{~mm}, \varepsilon=4.3$; (f) $\mathrm{hf}=1.2 \mathrm{~mm}, \varepsilon=8$. 


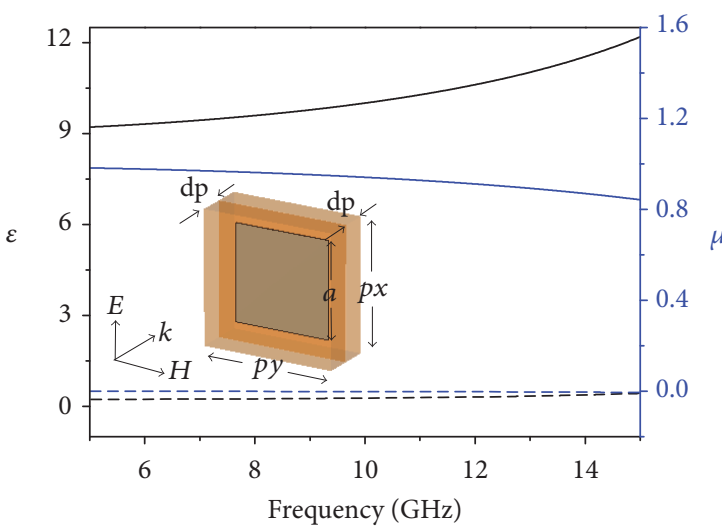

(a) Patch

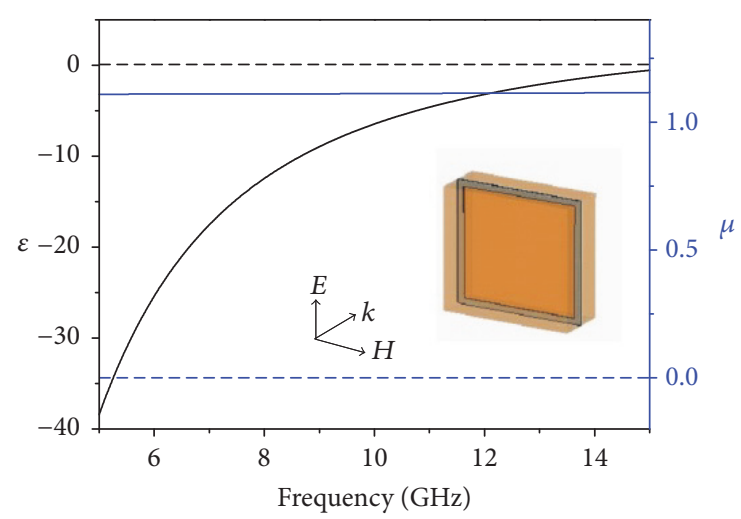

(b) Fishnet

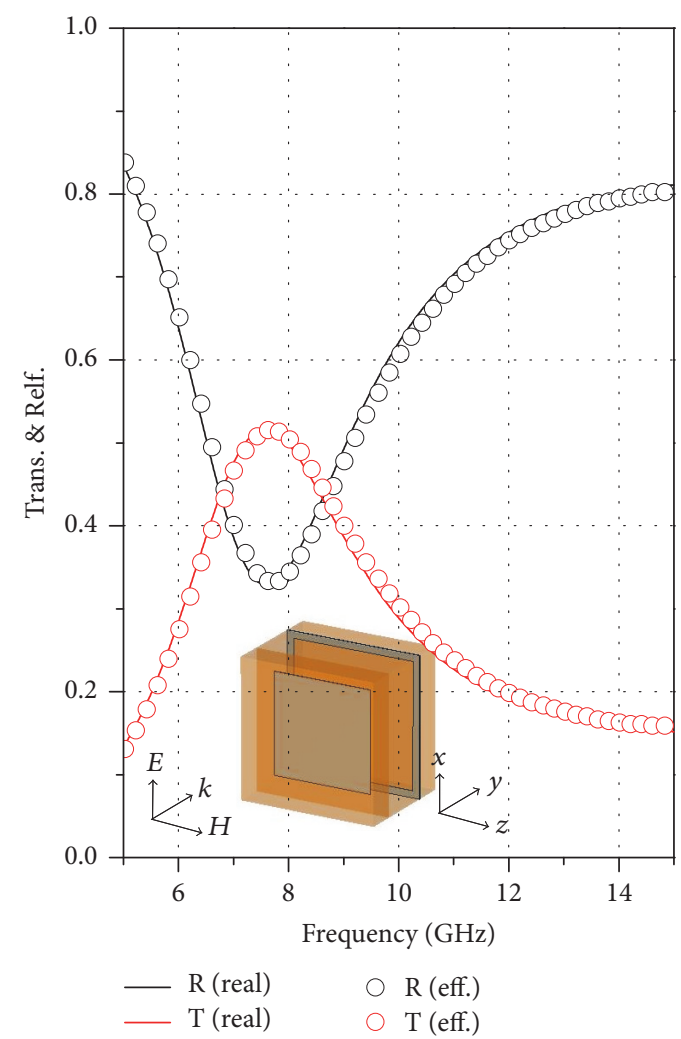

(c) Patch-fishnet

FIGURE 4: The calculated permittivity and permeability of symmetric (a) patch and (b) fishnet functional layers. (c) Transmission and reflection spectra of real patch-fishnet 2-functional-layer structure metamaterial (lines) and effective model (circles) under normal incidence.

symmetric functional layers with thicker dielectric layer have more robust effective parameters as we expected, since the coupling would be weaker in this case. On the other hand, thinner dielectric layer case is companied with strong coupling between metallic structures, and the robustness of effective parameters would be affected, as shown in Figure 3(c). However, compared to conventional fishnet structures with same spacer thickness between metallic structures (shown in Figure 3(d)), the robustness of effective parameters of symmetric functional layer system is still greatly improved in this case. The material parameters of dielectric spacer are another important factor that affected the coupling between metallic structures, since the damping of evanescent fields is scaled to the optical distance. We have also investigate a higher index spacer case $(\varepsilon=8$, blue); the thickness of dielectric layer is $\mathrm{df}=0.6 \mathrm{~mm}$. The optical distance between adjacent metallic layers in this case is nearly the same as in Figure 2(a). As shown in Figure 3(e), the obtained effective indexes are robust against the varying of the number of layers. This implies that more compact functional layers with 


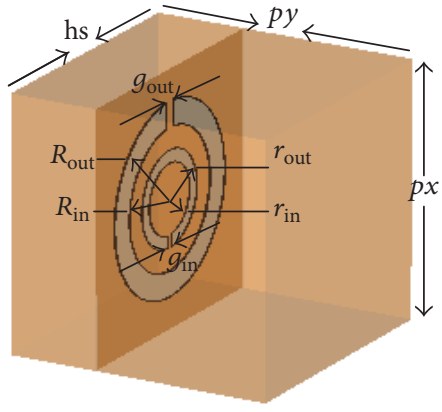

(a)

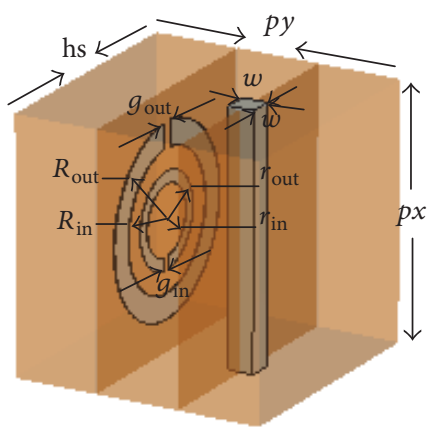

(d)

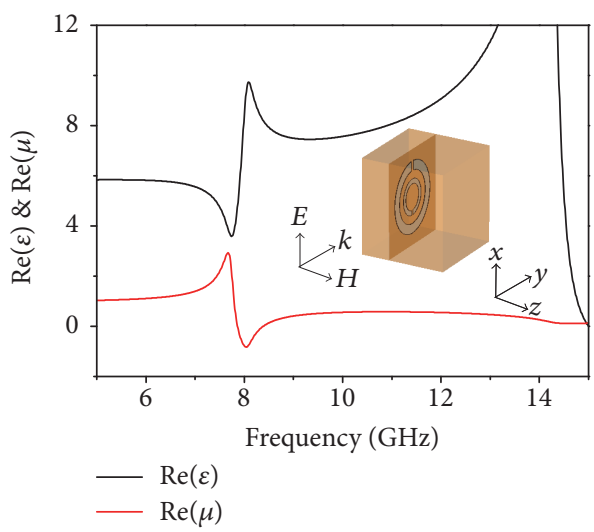

(b)

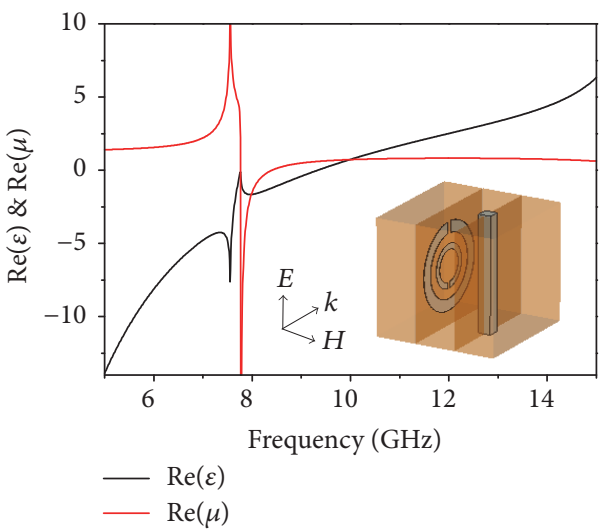

(e)

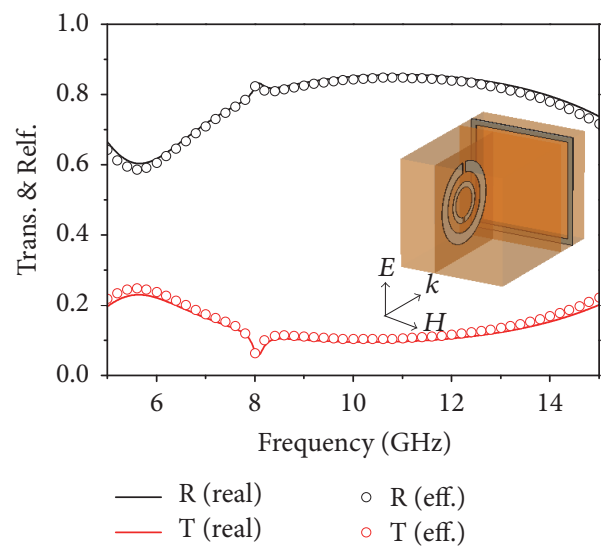

(c)

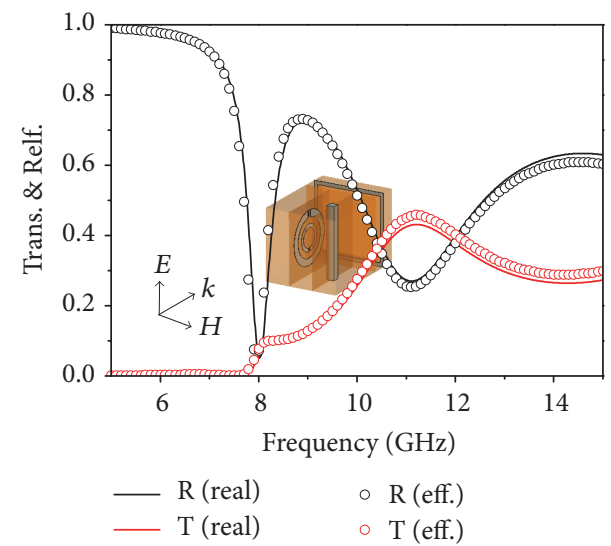

(f)

FIGURE 5: (a) and (d) Schematic of unit cells of symmetric SRR (a) and SRR/wire (d) functional layers. (b) and (e) The retrieved effective parameters for symmetric SRR (b) and SRR/wire (e) functional layers. (c) and (f) Transmission and reflection spectra of real SRR (SRR/wire) fishnet 2-functional-layer structure (lines) and effective model (circles).

robust effective parameters can be built with higher index spacer.

Since the symmetric functional layer design can significantly reduce the coupling between metallic structures, the robustness of effective parameters of symmetric functional layer should be maintained when different kinds of metal structures are used to construct multilayer MMs. In the insets of Figure 4(a), we present the unit cell of symmetric functional layer consisting of square patch structure. The length of patch is $a=2.9 \mathrm{~mm}$, the thickness of patch structures is $18 \mu \mathrm{m}$, and the periods along the lateral plane are the same as the aforementioned fishnet structure. FR4 layers with the thickness $\mathrm{dp}=0.8 \mathrm{~mm}$ are set on both sides of the metallic square patch. The symmetric fishnet functional layer shown in Figure 4(b) is exactly the same as the one in Figure 2(a). The effective parameters of patch and fishnet functional layers recovered from simulation data are shown in Figures 4(a) and 4(b). In principle, the imaginary parts of permittivity and permeability (black and blue dashed line) for both structures are rather small and can be neglected. The real parts of permeability of both slabs are near to 1 (nonmagnetic) [40].
For the patch structure, the real part (black solid line) of permittivity is positive, while it is negative for the fishnet (blue solid line). By stacking these two functional layers, we get a two-layer composite material; the unit cell is shown in the inset of Figure 4(c). A corresponding hypothetic material can be built by stacking two layers of homogeneous medium with the same thickness and recovered parameter of patch and fishnet functional layers. Considering an electromagnetic wave normal incidence to the real material and the hypothetical patch-fishnet slab, the transmission and reflection spectra are obtained by performing numerical simulation and transfer matrix method calculation, respectively. The results (see in Figure 4(c)) are almost identical to each other.

In addition, split ring resonators (SRRs) and SRR/wire structures $[2,3,9,13,14,16,19,26,32,33]$ are used to test the robustness of effective parameters of the symmetric functional layer. Figures 5(a) and 5(d) present the unit cell of symmetric functional layers consisting of SRR and SRR/wire structures. The SRR and SRR/wire structures are embedded in a FR4 dielectric layer with the thickness of $\mathrm{hs}=4 \mathrm{~mm}$, leaving the same distance from metal edge to 


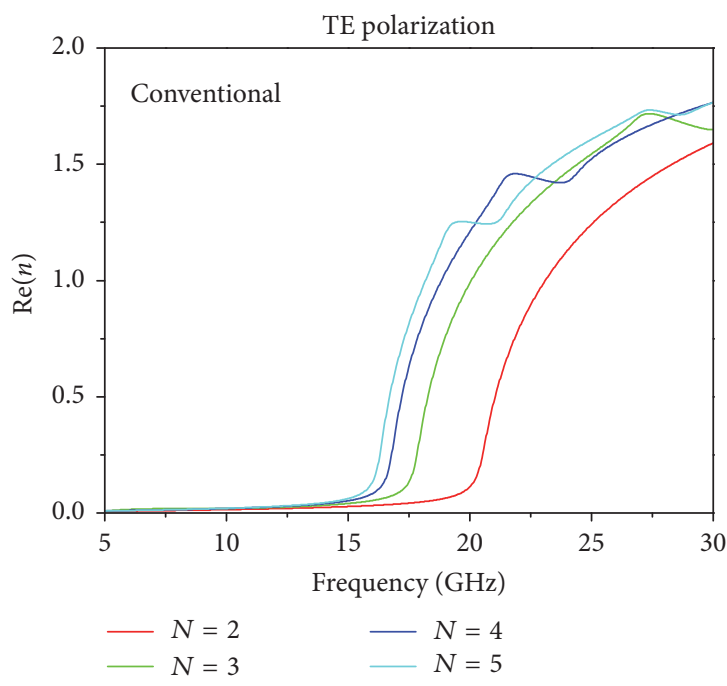

(a)

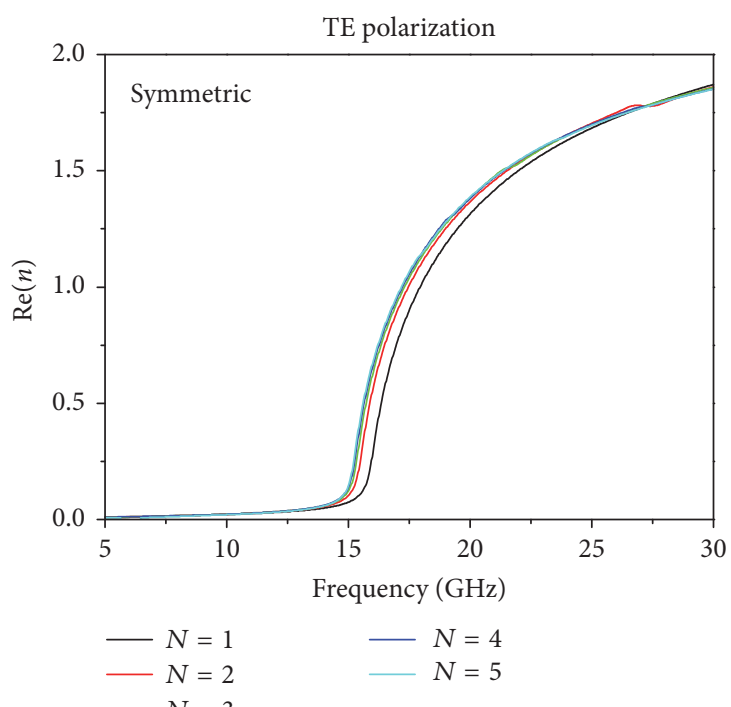

(c)

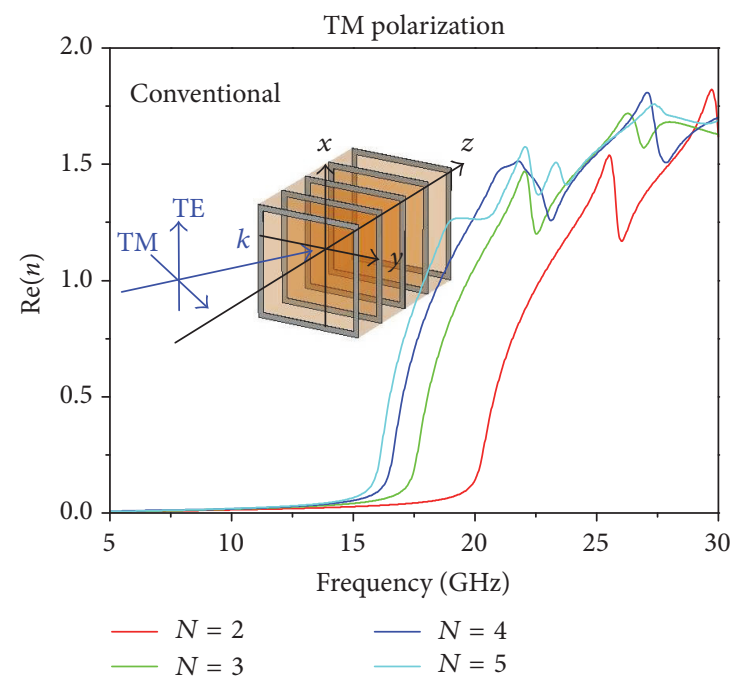

(b)

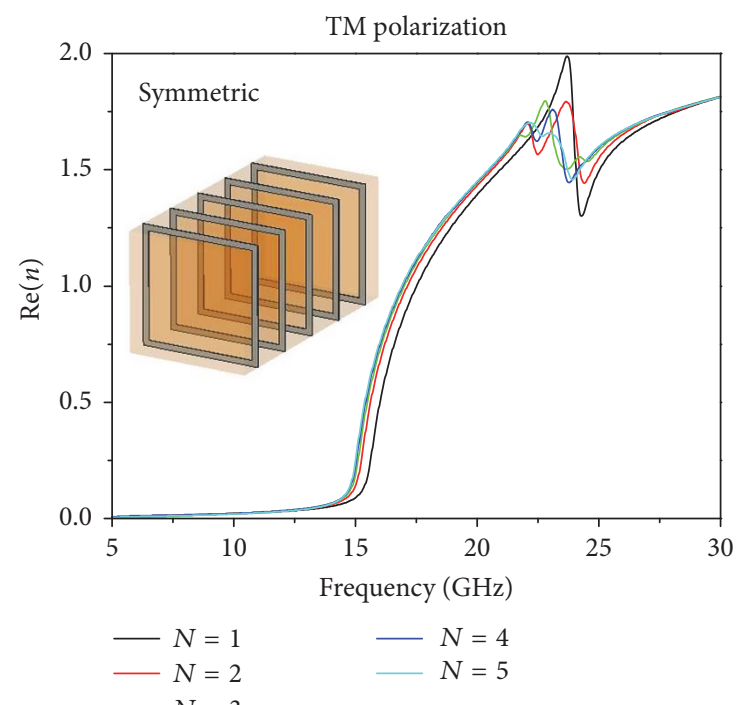

(d)

FIGURE 6: The definition of the polarization is shown in the inset of (b). Real part of effective index of conventional fishnet structures at 30-degree oblique incidence for TE (a) and TM (b) polarizations. The corresponding results of symmetric fishnet structures are shown in (c) and (d).

layer boundary on both sides. The lateral periods are $p x=$ $p y=4 \mathrm{~mm}$, same as the aforementioned fishnet and patch functional layers. The SRR structures have the thickness of $18 \mu \mathrm{m}$. The outer ring of SRR has outside radius of $R_{\text {out }}=$ $1.5 \mathrm{~mm}$, inside radius of $R_{\text {in }}=1.1 \mathrm{~mm}$, and gap of $g_{\text {out }}=$ $0.2 \mathrm{~mm}$; the inner one has outside radius of $r_{\text {out }}=0.75 \mathrm{~mm}$, inside radius of $r_{\text {in }}=0.55 \mathrm{~mm}$, and gap of $g_{\text {in }}=0.1 \mathrm{~mm}$. The width of the wire structure is $w=0.2 \mathrm{~mm}$ and it runs the length of the unit cell. As shown in Figures 5(b) and 5(e), effective permittivity and permeability of SRR and SRR/wire functional layers have strong resonant properties and exhibit magnetic negative and double negative features around the resonant frequencies. We pair the SRR (SRR/wire) functional layer with fishnet functional layer to form a two-layer slab; the unit cell is shown in the insets of Figures 5(c) and 5(f). The calculated transmission and reflection results of effective models match very well with those of the real structures (seen in Figures 5(c) and 5(f)), even around the resonant frequencies where the effective parameters rapidly change. Thus we conclude that symmetric functional layer design provides robust effective parameter and makes it possible to estimate macroscopic electromagnetic behavior of multilayer MMs based on effective parameter of all the included functional layers. Conversely, it also provides practical route to design multilayer MMs to be tailored for unprecedented applications. 


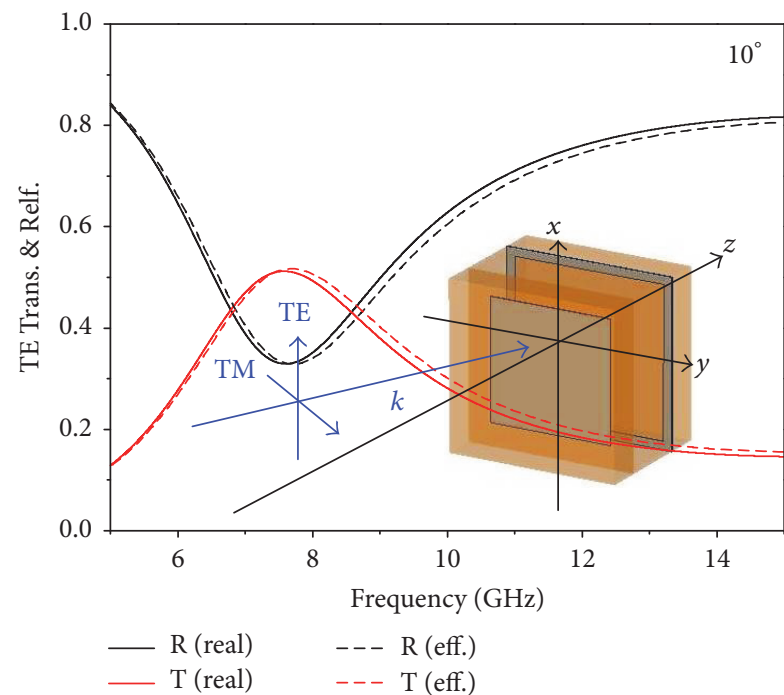

(a)
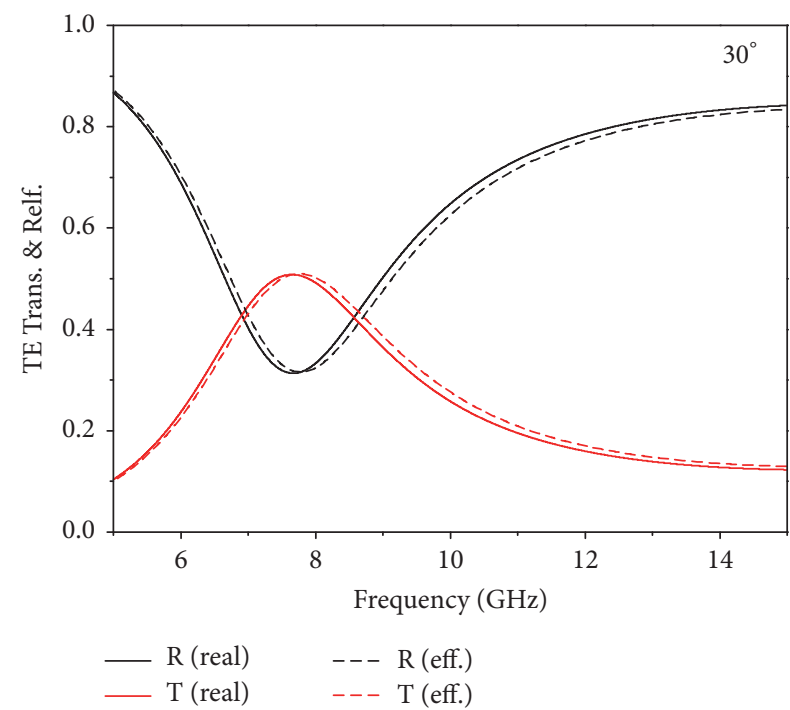

(c)

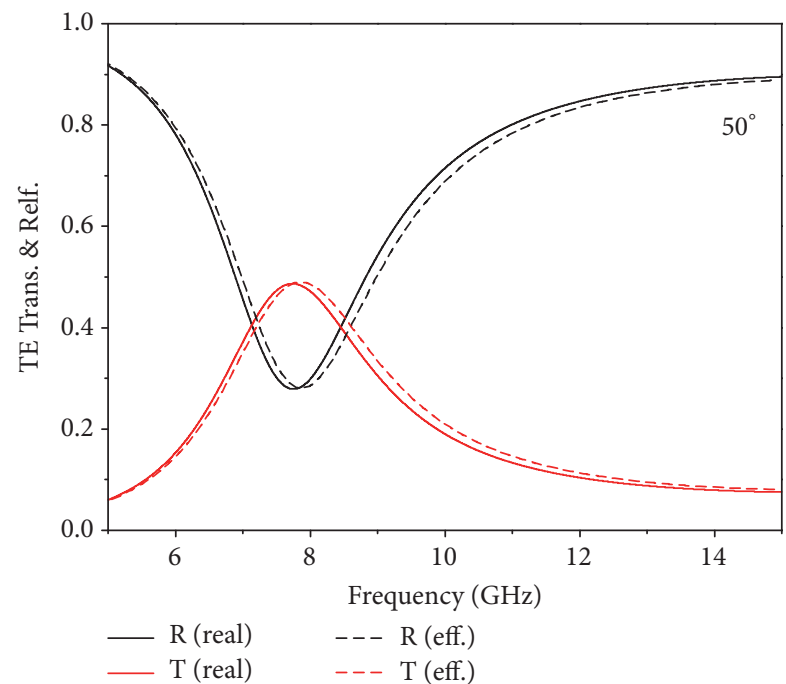

(e)

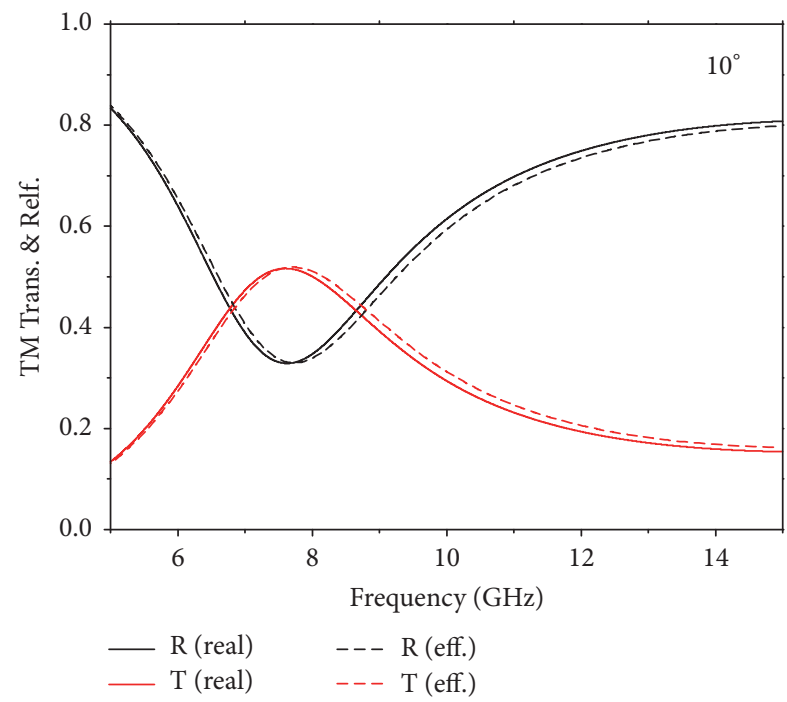

(b)

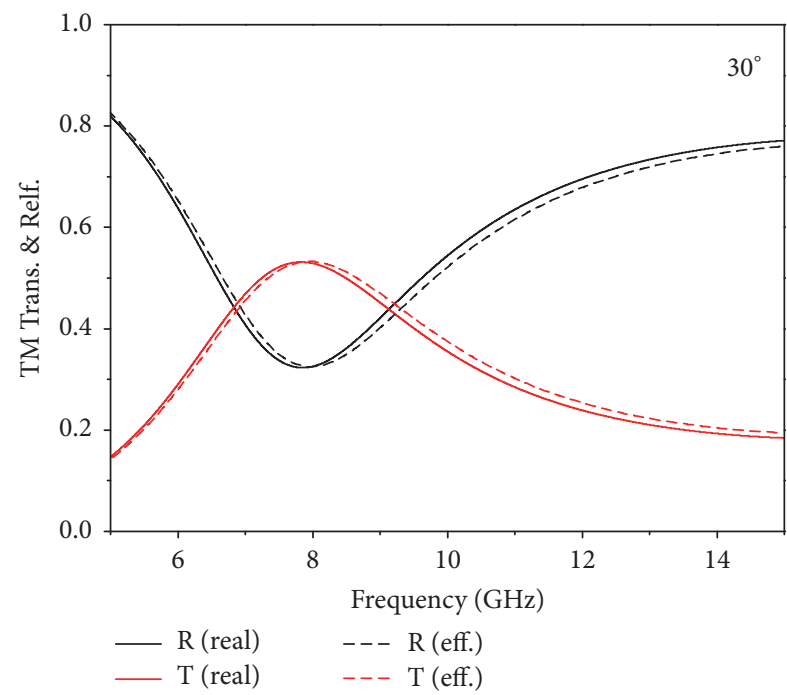

(d)

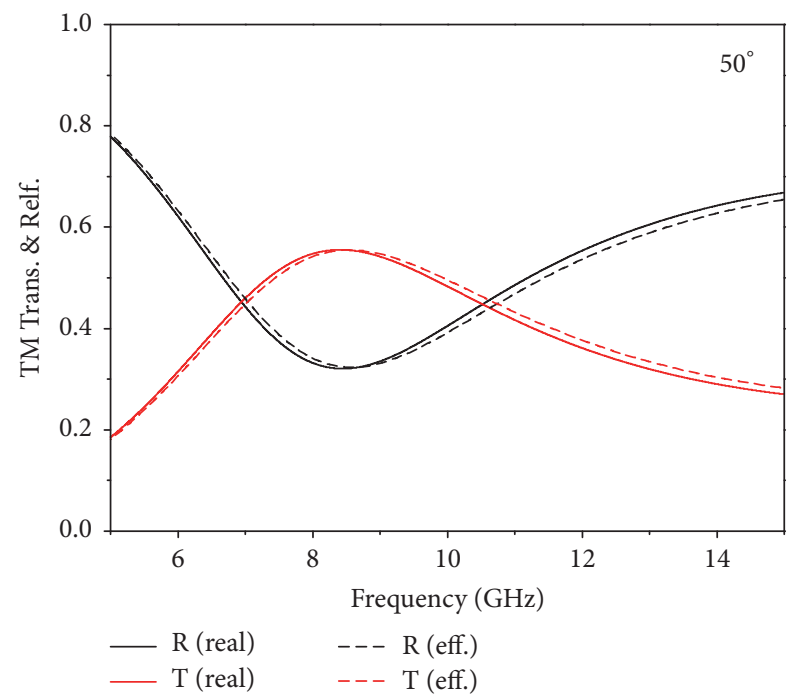

(f)

Figure 7: Continued. 


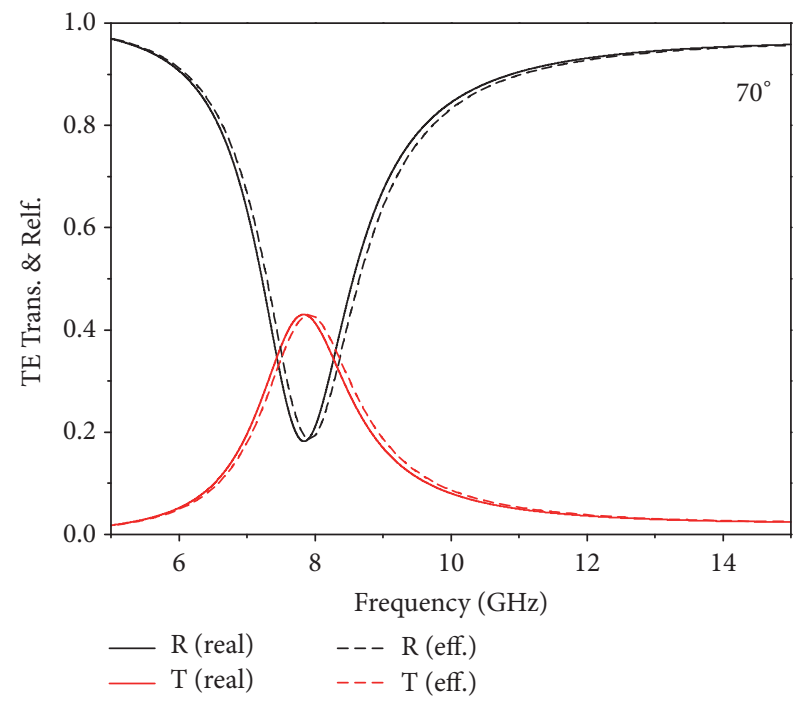

(g)

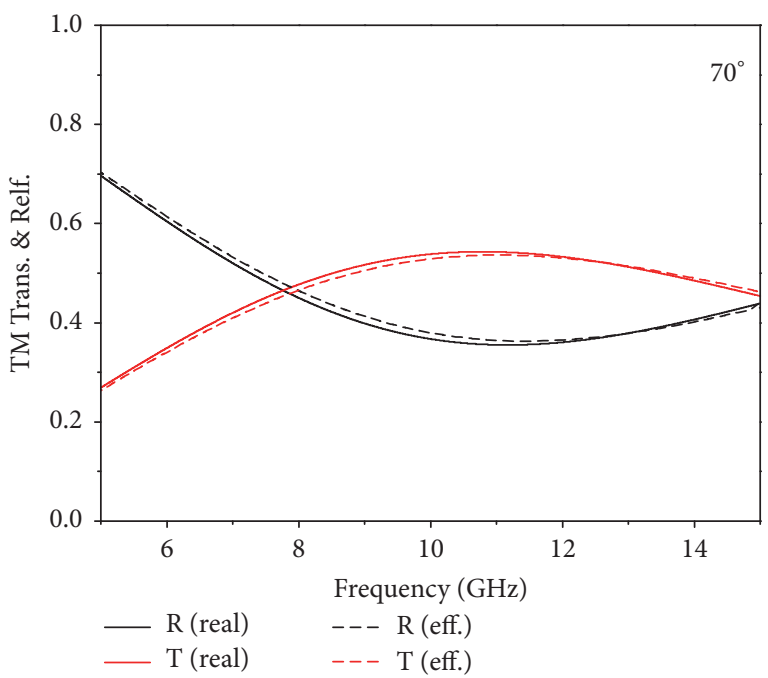

(h)

Figure 7: Transmission and reflection spectra of real patch-fishnet 2-layer structure (solid lines) and effective model (dashed lines) at oblique incidence for both polarizations.

\section{Oblique Incidence Case}

The implementation of MMs is not restricted to the normal incidence condition. Due to the specific shape and orientation of MM structures, effective parameters retrieved from the transmission and reflection coefficients of a MM slab are directly related to the incident fields. In principle, the retrieved complex effective parameters are not global material parameters. For instance, effective MM parameters for oblique incidence are determined by the incident angle and polarization, which are functions of the lateral wave vector [28].

For the oblique incidence case, the conventional designed multilayer MMs also have to accumulate enough layers to reach the convergence of effective parameters [28]. On the multilayer fishnet structures, we perform a comparison between the conventional design and the symmetric functional layer design. In Figure 6, we present retrieved results of the real part of effective index for both polarizations at 30 -degree incident angle. The plane of incidence is the $y-z$ plane, and the incident angle is defined by the angle between the $z$-axis and the wave vector $k$. The electric field vectors of both polarizations are denoted with blue arrows. Clearly, the robustness of effective parameters of symmetric functional layer is unaffected at oblique incidence situation for both polarizations. Especially, for the strongly resonance mode induced by the TM polarized incidence, symmetric functional layer design also provides robust effective parameters and avoids the hybridization in conventional design that affect the convergence in a wide frequency range.

Furthermore, we investigate the symmetric functional layer in multilayer MMs design and characterization at oblique incident case. If we try to build effective model of a multilayer MM for oblique incident case, the crux here is to determine the effective parameters for each slab. Considering a plane wave with an arbitrary angle of incidence enters a multilayer system, the lateral wave vector is preserved in all spatial regions. Therefore, a reasonable guess here is that each layer of the effective model should be homogenous medium with effective parameters retrieved for corresponding functional layers with the same lateral wave vector. However, it is kept in mind that this hypothetic effective model is only valid for multilayer MMs under this angle of incidence.

The robustness of effective parameters of symmetric functional layer is also tested with the patch-fishnet structure at oblique incidence case. The structure is exactly the same as the structure in Figure 4. Effective parameters of each functional layer are obtained at the same lateral wave vector of the system. Figure 7 presents the transmission and reflection spectra for effective model and the real structure at several different incident angles for both polarizations. Clearly, effective model is equivalent to the real structure for both polarizations in a wide range of incident angles.

SRR and SRR/wire structures are typical MMs building blocks, whose resonant features varied considerably as the incident angle increased. A SRR/wire-fishnet two-functionallayer structure under oblique incidence case is investigated to demonstrate the effective parameters robustness of symmetric functional layer design. The structure is exactly the same as in Figure 5(f). The transmission and reflection spectra for effective model and the real structure at the angle of incidence 30 and 60 degrees are shown in Figure 8. It is clear that symmetric ring resonator functional layers hold excellent robust effective parameters in oblique incidence cases and the electromagnetic behavior of real structure can be well characterized by effective model. 


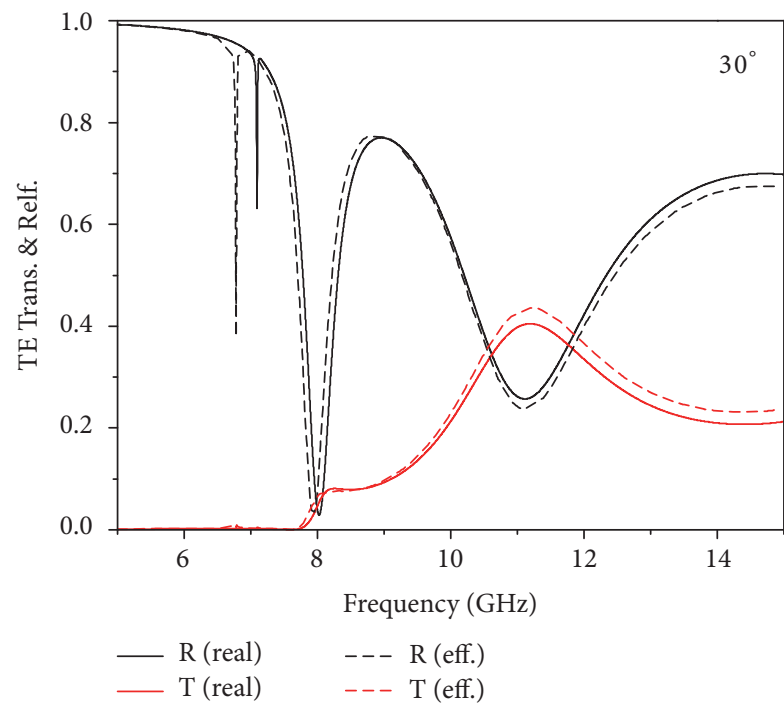

(a)

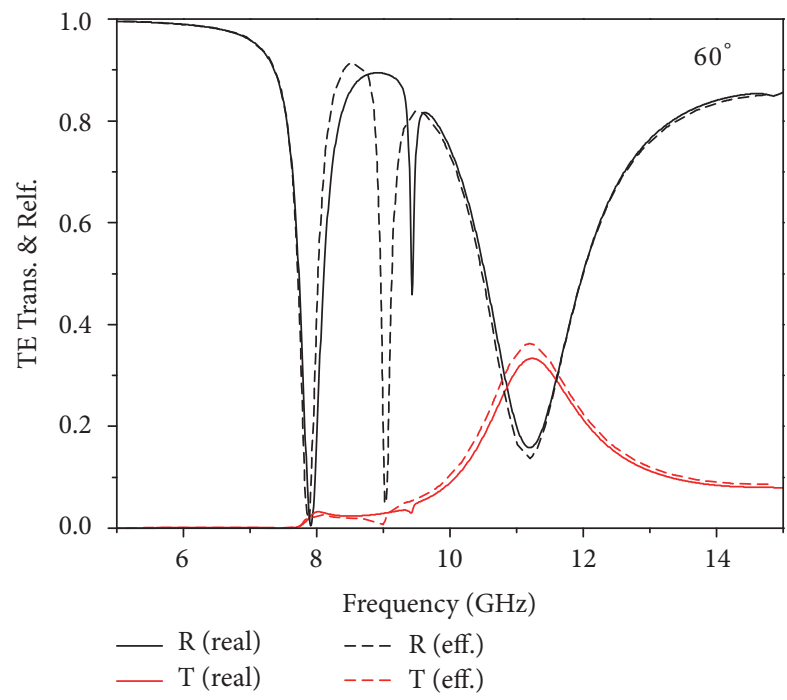

(c)

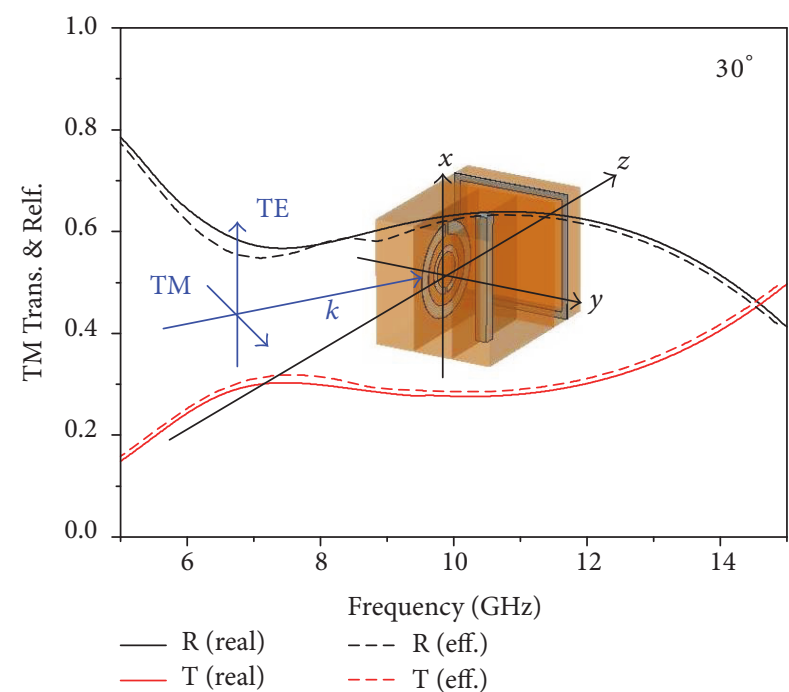

(b)

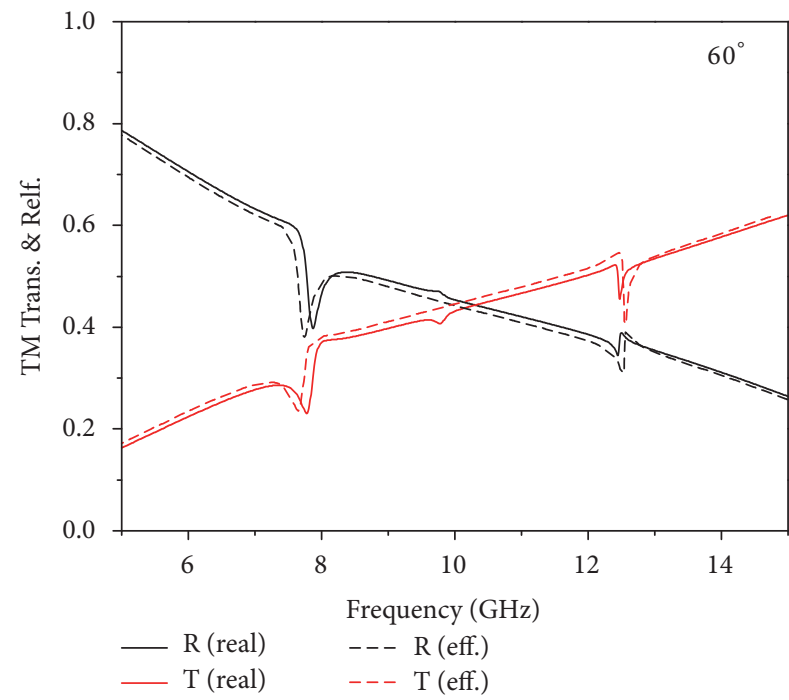

(d)

FIGURE 8: Transmission and reflection spectra of two-layer structure that is comprised of SRR/wire and fishnet functional-layer (solid lines) and the corresponding effective model (dashed lines) at oblique incidence $\left(30^{\circ}\right.$ and $\left.60^{\circ}\right)$ for both polarizations.

In addition, a three-functional-layer MM consisting of patch, SRR/wire, and fishnet structures is investigated here to give further demonstration. Each functional layer here has the same geometric parameters as those aforementioned. In Figure 9, we plot the transmission and reflection results for both polarizations at the angle of incidence of 30 and 60 degrees. Effective parameters of symmetric functional layer still show great robustness in such complex multilayer structures. The effective model results match pretty well with those of real system through the frequency range we investigated, especially for the TM polarization. The SRR/wire structures have strong resonance of electric field for the TE polarization, which causes the frequency shifting at the resonance band with the increasing angle of incidence. But still, the effective model shows great equivalency to the real structure.

\section{Conclusion}

We have proposed a symmetric functional layer design with robust effective parameters for multilayer MMs. The robustness of effective parameters of symmetric functional layer are examined in multilayer MMs comprising several layers of different kinds of metallic building blocks at both normal and oblique incident cases. In general, the symmetric functional layer provides the opportunity to straightforwardly design and characterize the electromagnetic properties of multilayer MMs by effective parameters. 


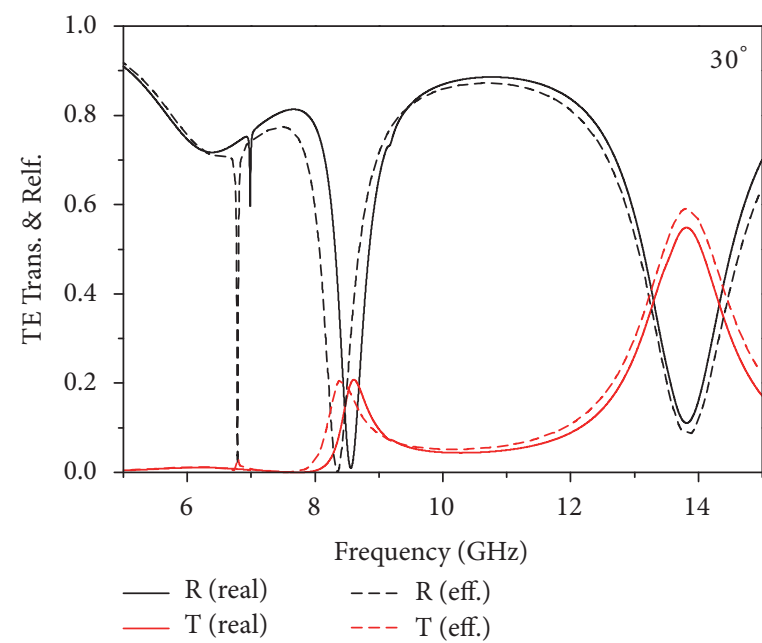

(a)

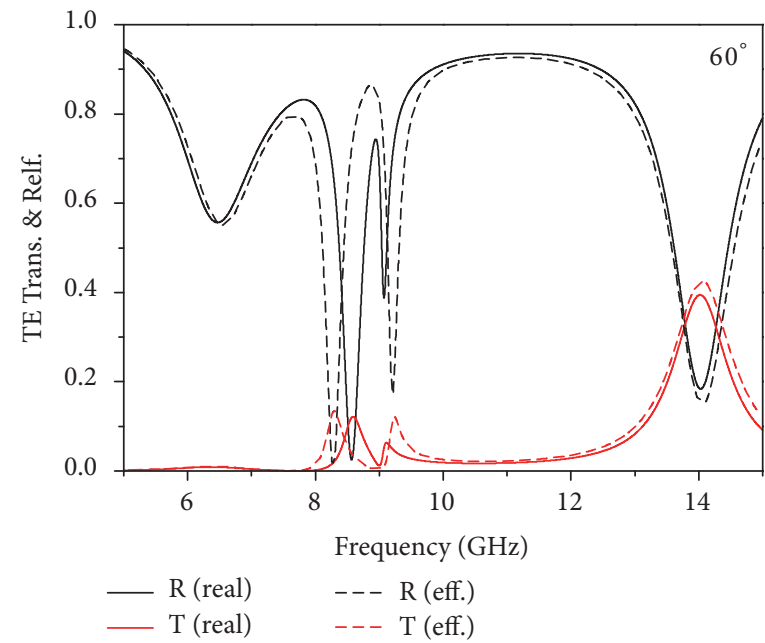

(c)

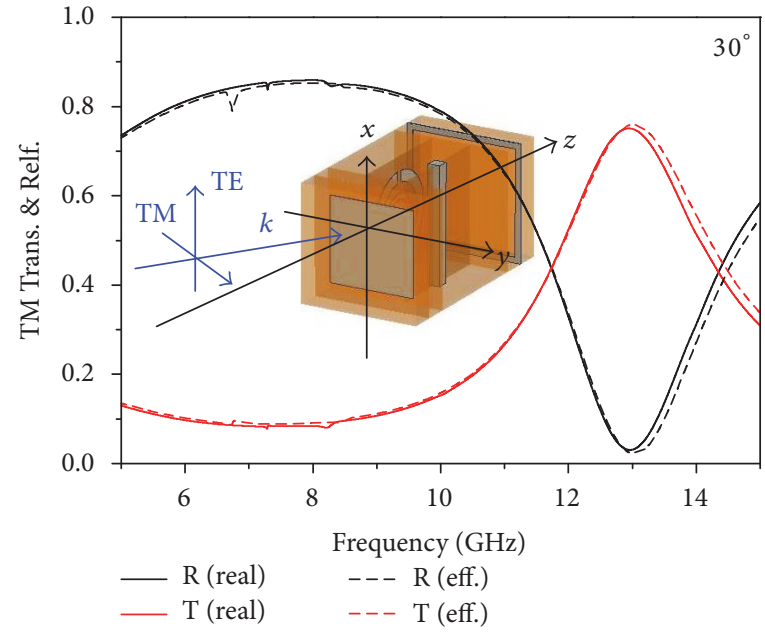

(b)

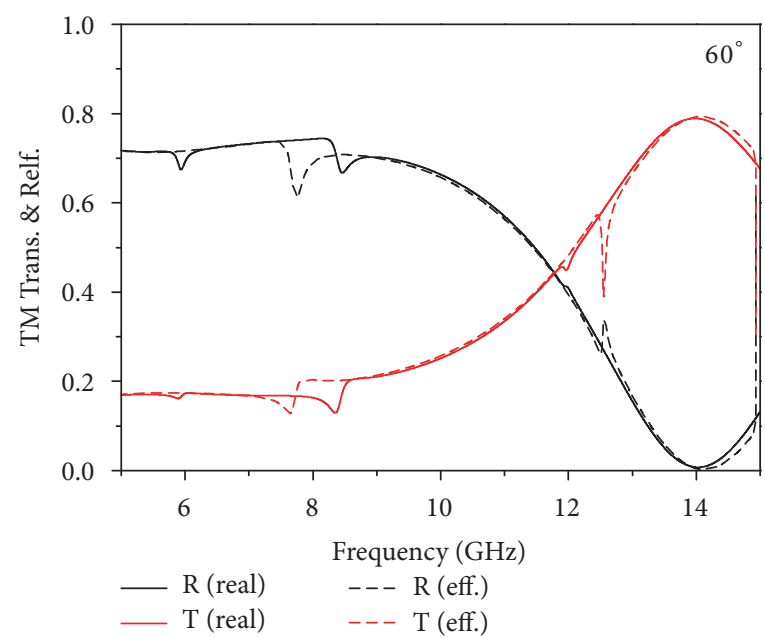

(d)

FIGURE 9: Transmission and reflection spectra of three-layer structure that is comprised of patch, SRR/wire and fishnet functional layer (solid lines), and the corresponding effective model (dashed lines) at oblique incidence $\left(30^{\circ}\right.$ and $\left.60^{\circ}\right)$ for both polarizations.

\section{Conflicts of Interest}

The authors declare that there are no conflicts of interest regarding the publication of this paper.

\section{Acknowledgments}

This work was supported by the National Natural Science Foundation of China (Grant nos. 11174221, 11204218, 11404213, 61205041, and 11674248) and the China Postdoctoral Science Foundation (Grant nos. 2013T60460 and 2012M520922).

\section{References}

[1] J. B. Pendry, A. J. Holden, W. J. Stewart, and I. Youngs, "Extremely low frequency plasmons in metallic mesostructures," Physical Review Letters, vol. 76, p. 4773, 1996.

[2] J. B. Pendry, A. J. Holden, D. J. Robbins, and W. J. Stewart, "Magnetism from conductors and enhanced nonlinear phenomena,"
IEEE Transactions on Microwave Theory and Techniques, vol. 47, no. 11, pp. 2075-2084, 1999.

[3] R. A. Shelby, D. R. Smith, and S. Schultz, "Experimental verification of a negative index of refraction," Science, vol. 292, no. 5514, pp. 77-79, 2001.

[4] J. B. Pendry, "Negative refraction makes a perfect lens," Physical Review Letters, vol. 85, no. 18, pp. 3966-3969, 2000.

[5] J. B. Pendry, D. Schurig, and D. R. Smith, "Controlling electromagnetic fields," Science, vol. 312, no. 5781, pp. 1780-1782, 2006.

[6] N. I. Landy, S. Sajuyigbe, J. J. Mock, D. R. Smith, and W. J. Padilla, "Perfect metamaterial absorber," Physical Review Letters, vol. 100, no. 20, Article ID 207402, 2008.

[7] C. M. Soukoulis and M. Wegener, "Past achievements and future challenges in the development of three-dimensional photonic metamaterials," Nature Photonics, vol. 5, no. 9, pp. 523-530, 2011.

[8] G. Dolling, M. Wegener, and S. Linden, "Realization of a threefunctional-layer negative-index photonic metamaterial," Optics Letters, vol. 32, no. 5, pp. 551-553, 2007. 
[9] N. Liu, H. Guo, L. Fu, S. Kaiser, H. Schweizer, and H. Giessen, "Three-dimensional photonic metamaterials at optical frequencies," Nature Materials, vol. 7, no. 1, pp. 31-37, 2008.

[10] J. Valentine, S. Zhang, T. Zentgraf et al., “Three-dimensional optical metamaterial with a negative refractive index," Nature, vol. 455, no. 7211, pp. 376-379, 2008.

[11] E. Plum, J. Zhou, J. Dong et al., "Metamaterial with negative index due to chirality," Physical Review B, vol. 79, no. 3, Article ID 035407, 2009.

[12] J. Zhou, T. Koschny, M. Kafesaki, and C. M. Soukoulis, "Negative refractive index response of weakly and strongly coupled optical metamaterials," Physical Review B, vol. 80, no. 3, Article ID 035109, 2009.

[13] M. Decker, R. Zhao, C. M. Soukoulis, S. Linden, and M. Wegener, "Twisted split-ring-resonator photonic metamaterial with huge optical activity," Optics Letters, vol. 35, no. 10, pp. 1593-1595, 2010.

[14] F. Miyamaru, S. Kuboda, K. Taima, K. Takano, M. Hangyo, and M. W. Takeda, "Three-dimensional bulk metamaterials operating in the terahertz range," Applied Physics Letters, vol. 96, no. 8, Article ID 081105, 2010.

[15] F. Ding, Y. Cui, X. Ge, Y. Jin, and S. He, "Ultra-broadband microwave metamaterial absorber," Applied Physics Letters, vol. 100, no. 10, Article ID 103506, 2012.

[16] N. Katsarakis, G. Konstantinidis, A. Kostopoulos et al., "Magnetic response of split-ring resonators in the far-infrared frequency regime," Optics Letters, vol. 30, no. 11, pp. 1348-1350, 2005.

[17] A. Boltasseva and V. M. Shalaev, "Fabrication of optical negative-index metamaterials: recent advances and outlook," Metamaterials, vol. 2, no. 1, pp. 1-17, 2008.

[18] M. Decker, M. Ruther, C. E. Kriegler et al., "Strong optical activity from twisted-cross photonic metamaterials," Optics Letters, vol. 34, no. 16, pp. 2501-2503, 2009.

[19] X. Xiong, W.-H. Sun, and Y.-J. Bao, "Construction of a chiral metamaterial with a U-shaped resonator assembly," Physical Review B, vol. 81, no. 7, Article ID 075119, 6 pages, 2010.

[20] J. Zhou, R. Zhao, C. M. Soukoulis, A. J. Taylor, and J. O’Hara, "Chiral $\mathrm{THz}$ metamaterial with tunable optical activity," in Proceedings of the Conference on Lasers and Electro-Optics, CTuF, San Jose, Calif, USA, May 2010.

[21] D. B. Burckel, J. R. Wendt, G. A. Ten Eyck et al., "Micrometerscale cubic unit cell 3D metamaterial layers," Advanced Materials, vol. 22, no. 44, pp. 5053-5057, 2010.

[22] D. Ö. Güney, T. Koschny, and C. M. Soukoulis, "Intra-connected three-dimensionally isotropic bulk negative index photonic metamaterial," Optics Express, vol. 18, no. 12, pp. 12348-12353, 2010.

[23] J. Fischer, G. Von Freymann, and M. Wegener, "The materials challenge in diffraction-unlimited dired-laser-writing optical lithography," Advanced Materials, vol. 22, no. 32, pp. 3578-3582, 2010.

[24] M. Deubel, G. von Freymann, M. Wegener, S. Pereira, K. Busch, and C. M. Soukoulis, "Direct laser writing of three-dimensional photonic-crystal templates for telecommunications," Nature Materials, vol. 3, no. 7, pp. 444-447, 2004.

[25] J. K. Gansel, M. Thiel, M. S. Rill et al., "Gold helix photonic metamaterial as broadband circular polarizer," Science, vol. 325, no. 5947, pp. 1513-1515, 2009.

[26] D. R. Smith, S. Schultz, P. Markoš, and C. M. Soukoulis, "Determination of effective permittivity and permeability of metamaterials from reflection and transmission coefficients," Physical Review B, vol. 65, Article ID 195104, 2002.

[27] D. R. Smith, D. C. Vier, T. Koschny, and C. M. Soukoulis, "Electromagnetic parameter retrieval from inhomogeneous metamaterials," Physical Review E, vol. 71, no. 3, Article ID $036617,2005$.

[28] C. Menzel, C. Rockstuhl, T. Paul, F. Lederer, and T. Pertsch, "Retrieving effective parameters for metamaterials at oblique incidence," Physical Review B, vol. 77, Article ID 195328, 2008.

[29] M. C. K. Wiltshire, J. B. Pendry, and J. V. Hajnal, "Chiral Swiss rolls show a negative refractive index," Journal of Physics Condensed Matter, vol. 21, no. 29, Article ID 292201, 2009.

[30] S. Zhang, Y.-S. Park, J. Li, X. Lu, W. Zhang, and X. Zhang, "Negative refractive index in chiral metamaterials," Physical Review Letters, vol. 102, no. 2, Article ID 023901, 2009.

[31] J. Zhou, J. Dong, B. Wang, T. Koschny, M. Kafesaki, and C. M. Soukoulis, "Negative refractive index due to chirality," Physical Review B, vol. 79, no. 12, Article ID 121104, 2009.

[32] X. Chen, B.-I. Wu, J. A. Kong, and T. M. Grzegorczyk, "Retrieval of the effective constitutive parameters of bianisotropic metamaterials," Physical Review E, vol. 71, Article ID 046610, 2005.

[33] Z. Li, K. Aydin, and E. Ozbay, "Determination of the effective constitutive parameters of bianisotropic metamaterials from reflection and transmission coefficients," Physical Review E, vol. 79, no. 2, Article ID 026610, 2009.

[34] Z. Li, R. Zhao, T. Koschny et al., "Chiral metamaterials with negative refractive index based on four ' $U$ ' split ring resonators," Applied Physics Letters, vol. 97, no. 8, Article ID 081901, 2010.

[35] R. Zhao, T. Koschny, and C. M. Soukoulis, "Chiral metamaterials: retrieval of the effective parameters with and without substrate," Optics Express, vol. 18, no. 14, pp. 14553-14567, 2010.

[36] S. Zhang, W. Fan, N. C. Panoiu, K. J. Malloy, R. M. Osgood, and S. R. J. Brueck, "Experimental demonstration of near-infrared negative-index metamaterials," Physical Review Letters, vol. 95, no. 13, Article ID 137404, 2005.

[37] J. Tuma, O. Lyutakov, I. Goncharova, and V. Svorcik, "AgPMMA structures for application in infra-red optical range," Materials Chemistry and Physics, vol. 148, no. 1-2, pp. 343-348, 2014.

[38] Y. Kalachyova, O. Lyutakov, A. Solovyev, P. Slepička, and V. Švorčík, "Surface morphology and optical properties of porphyrin/Au and Au/porphyrin/Au systems," Nanoscale Research Letters, vol. 8, no. 1, pp. 1-23, 2013.

[39] X. Chen, T. M. Grzegorczyk, B.-I. Wu, J. Pacheco Jr., and J. A. Kong, "Robust method to retrieve the constitutive effective parameters of metamaterials," Physical Review E, vol. 70, Article ID 016608, 2004.

[40] L. D. Landau, J. Bell, M. Kearsley, L. Pitaevskii, E. Lifshitz, and J. Sykes, Electrodynamics of Continuous Media, vol. 8 of Elsevier, Amsterdam, Netherlands, 1984. 

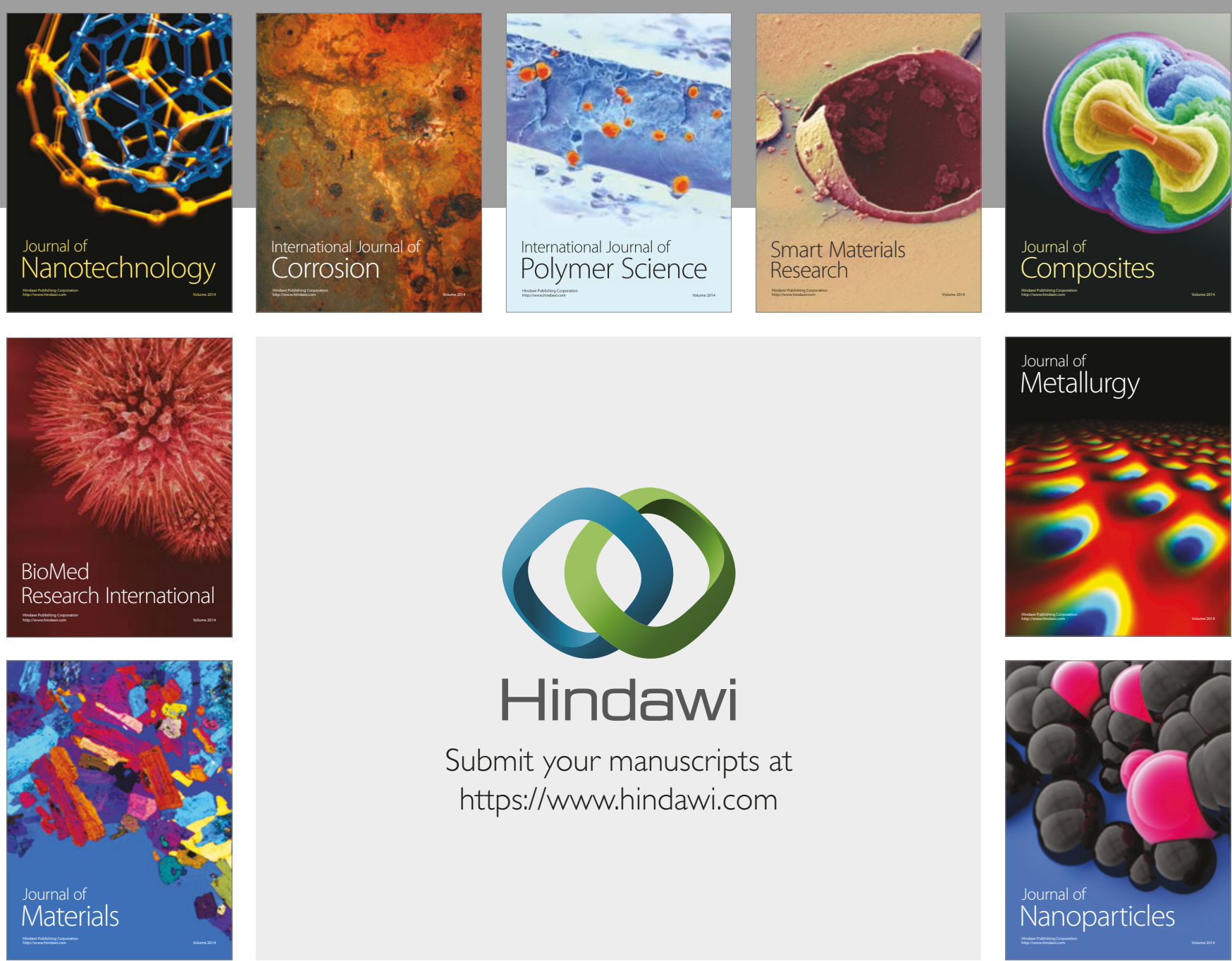

\section{Hindawi}

Submit your manuscripts at

https://www.hindawi.com
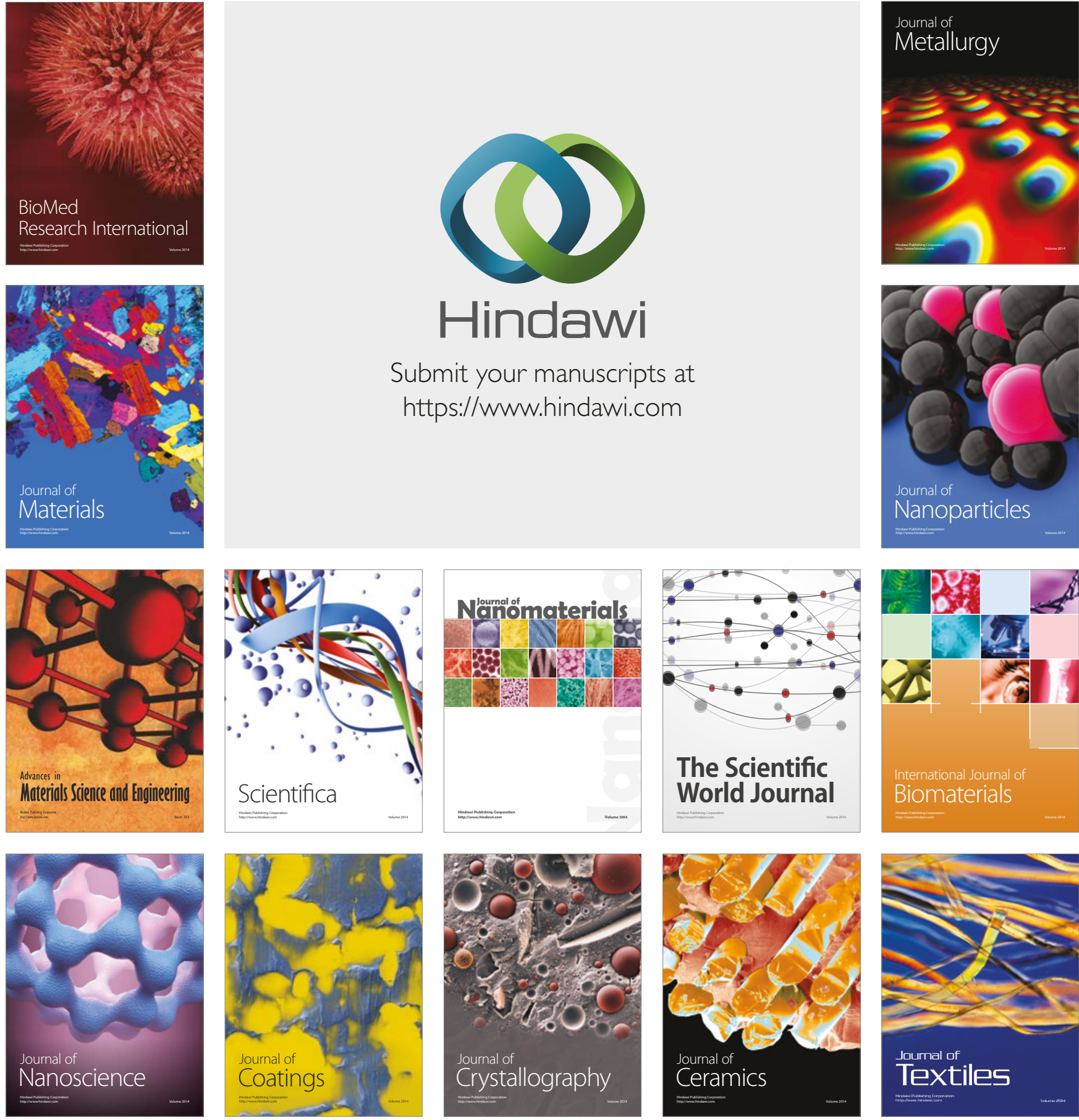

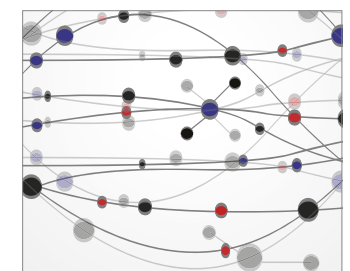

The Scientific World Journal
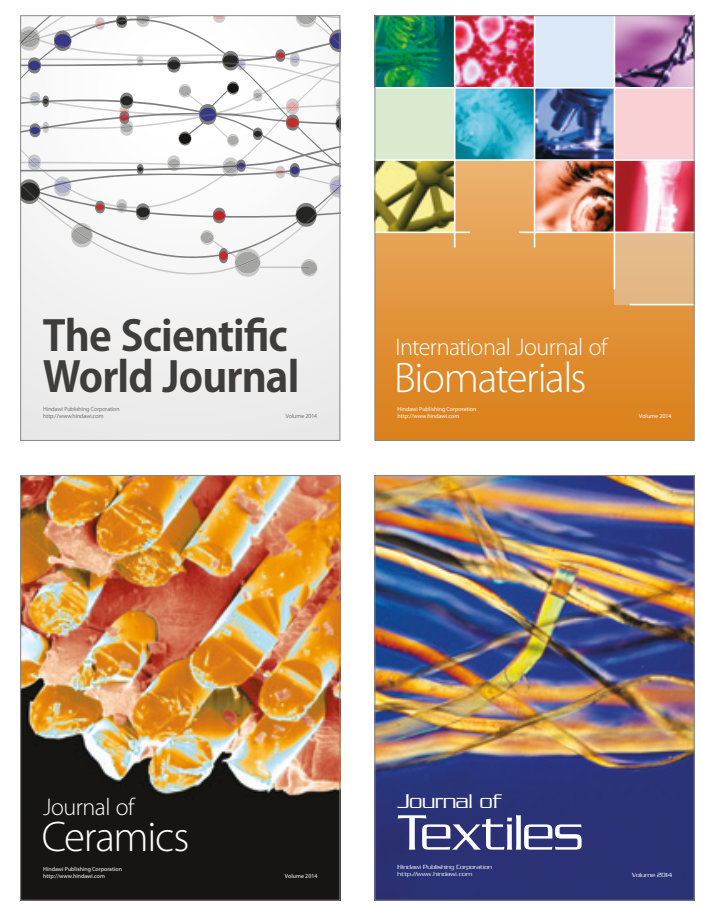